Stephanie M. W. Y. van de Ven

Sjoerd G. Elias

Andrea J. Wiethoff

Marjolein van der Voort

Tim Nielsen

Bernhard Brendel

Claas Bontus

Falk Uhlemann

Rami Nachabe

Rik Harbers

Michiel van Beek

Leon Bakker

Martin B. van der Mark

Peter Luijten

Willem P. Th. M. Mali

\section{Diffuse optical tomography of the breast: preliminary findings of a new prototype and comparison with magnetic resonance imaging}

Received: 4 July 2008

Revised: 14 November 2008

Accepted: 27 November 2008

Published online: 10 January 2009

(C) The Author(s) 2008.

This article is published with open access at

Springerlink.com

S. M. W. Y. van de Ven $(\bowtie)$.

S. G. Elias · P. Luijten ·

W. P. T. M. Mali

Department of Radiology,

University Medical Center Utrecht,

E.01.132, Heidelberglaan 100,

P.O. Box 85500,3508

GA Utrecht, The Netherlands

e-mail: s.m.w.y.vandeven-

2@umcutrecht.nl

Tel.: +31-88-7556687

Fax: +31-30-2581098

A. J. Wiethoff · R. Nachabe

Philips Healthcare,

Best, The Netherlands

M. van der Voort - R. Harbers .

M. van Beek · L. Bakker .

M. B. van der Mark

Philips Research Europe,

Eindhoven, The Netherlands
T. Nielsen · B. Brendel - C. Bontus ·

F. Uhlemann

Philips Research Europe,

Hamburg, Germany

Abstract This paper presents an evaluation of a prototype diffuse optical tomography (DOT) system. Seventeen women with 18 breast lesions (10 invasive carcinomas, 2 fibroadenomas, and 6 benign cysts; diameters $13-54 \mathrm{~mm}$ ) were evaluated with DOT and magnetic resonance imaging (MRI). A substantial fraction of the original 36 recruited patients could not be examined using this prototype due to technical problems. A region of interest (ROI) was drawn at the lesion position as derived from MRI and at the mirror image site in the contralateral healthy breast. ROIs were assessed quantitatively and qualitatively by two observers independently in two separate readings. Intra- and interobserver agreements were calculated using kappa statistics $(k)$ and intraclass correlation coefficients (ICCs). Discriminatory values for presence of malignancy were determined by receiver operating characteristic (ROC) analyses. Intraobserver agreements were excellent $(k 0.88$ and 0.88 ; ICC 0.978 and 0.987 ), interobserver agreements were good to excellent $(k$ 0.77-0.95; ICC 0.96-0.98). Discriminatory values for presence of malignancy were $0.92-0.93$ and $0.97-0.99$ for quantitative and qualitative ROC analysis, respectively. This DOT system has the potential to discriminate malignant from benign breast tissue in a reproducible qualitative and quantitative manner. Important technical improvements are required before this technique is ready for clinical application.

Keywords Optical tomography . Absorption - Breast cancer - Breast imaging

\section{Introduction}

Diffuse optical tomography (DOT) employs light in the near-infrared (NIR) spectrum, where light absorption is minimal (650-1,000 nm), to assess the optical properties of tissue [1]. Information on the composition of the breast tissue, i.e., relative concentrations of oxy- and deoxyhemoglobin, water, and lipid can be obtained by combining images acquired at different wavelengths (spectroscopic imaging). Hemoglobin concentration in a malignant tumor is directly related to angiogenesis [2], and proportions of oxy- and deoxyhemoglobin change as a result of its metabolism [3]. By using multiple wavelengths to measure concentrations of the main chromophores in the breast, discrimination between benign and malignant tissue may be possible with DOT. This technique could potentially have added value to currently used breast imaging modalities, i.e., mammography, ultrasound, and dynamic 


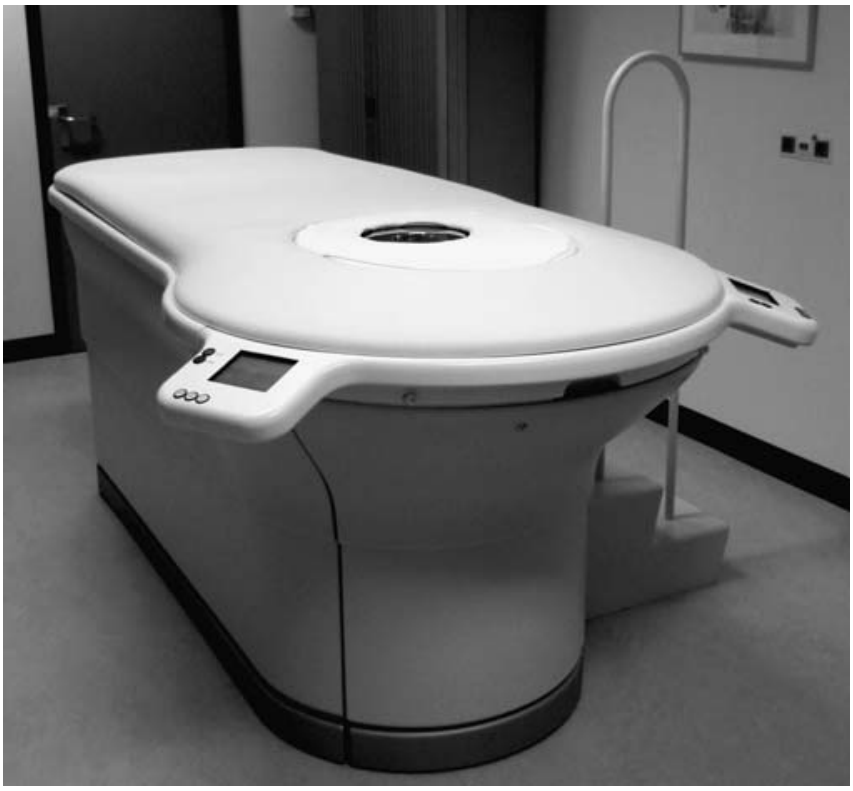

Fig. 1 Diffuse optical tomography prototype (Philips Healthcare, Best, The Netherlands)

contrast-enhanced magnetic resonance imaging (DCEMRI), which all have drawbacks regarding sensitivity and specificity in the diagnosis, early detection, and treatment monitoring of breast cancer [4-7].

Since optical breast imaging is still in its infancy with several techniques being explored [8], thorough system evaluation and validation is essential. We used MRI as a benchmark for the first diagnostic evaluation of our new prototype since it provides three-dimensional data and excellent soft tissue contrast. We initiated the evaluation under optimal settings in a small patient group by (1) investigating optical properties of different types of breast lesions; (2) assessing the potential to discriminate between benign and malignant tissue with a known lesion position; and (3) assessing the effect of intra- and interobserver variability on the obtained results.

\section{Methods}

\section{Patients}

Seventeen women (mean age 54, range 22-85) diagnosed with one or more breast lesion(s) 13 to $54 \mathrm{~mm}$ in diameter were prospectively included between August 2006 and September 2007 from the University Medical Center Utrecht and the Diakonessenhuis Utrecht, The Netherlands. Patients were referred either by their family doctor or breast surgeon for diagnostic purposes, or via the screening program for further workup. Patients were asked to participate in our study if a BI-RADS (Breast Imaging Reporting and Data System) 2-5 lesion was diagnosed on mammography/ultrasound, and if needle aspiration was not performed before the other study procedures could be executed, since this could influence the optical images. All patients underwent optical imaging and DCE-MRI. In total, 36 patients were recruited consecutively; however, 19 of
Fig. 2 Examples of ROIs on DOT images compared with MRI. a Patient is a 60 -year-old woman with lesion in the right breast; BI-RADS category 2 on MRI (left image T2-weighted MRI with fat suppression); visibility score -4 on DOT (right image); final diagnosis, benign cyst. b Patient is a 56-year-old woman with lesion in the right breast; BI-RADS category 5 on MRI (left image DCE-MRI); visibility score +4 on DOT (right image); final diagnosis, invasive ductal carcinoma
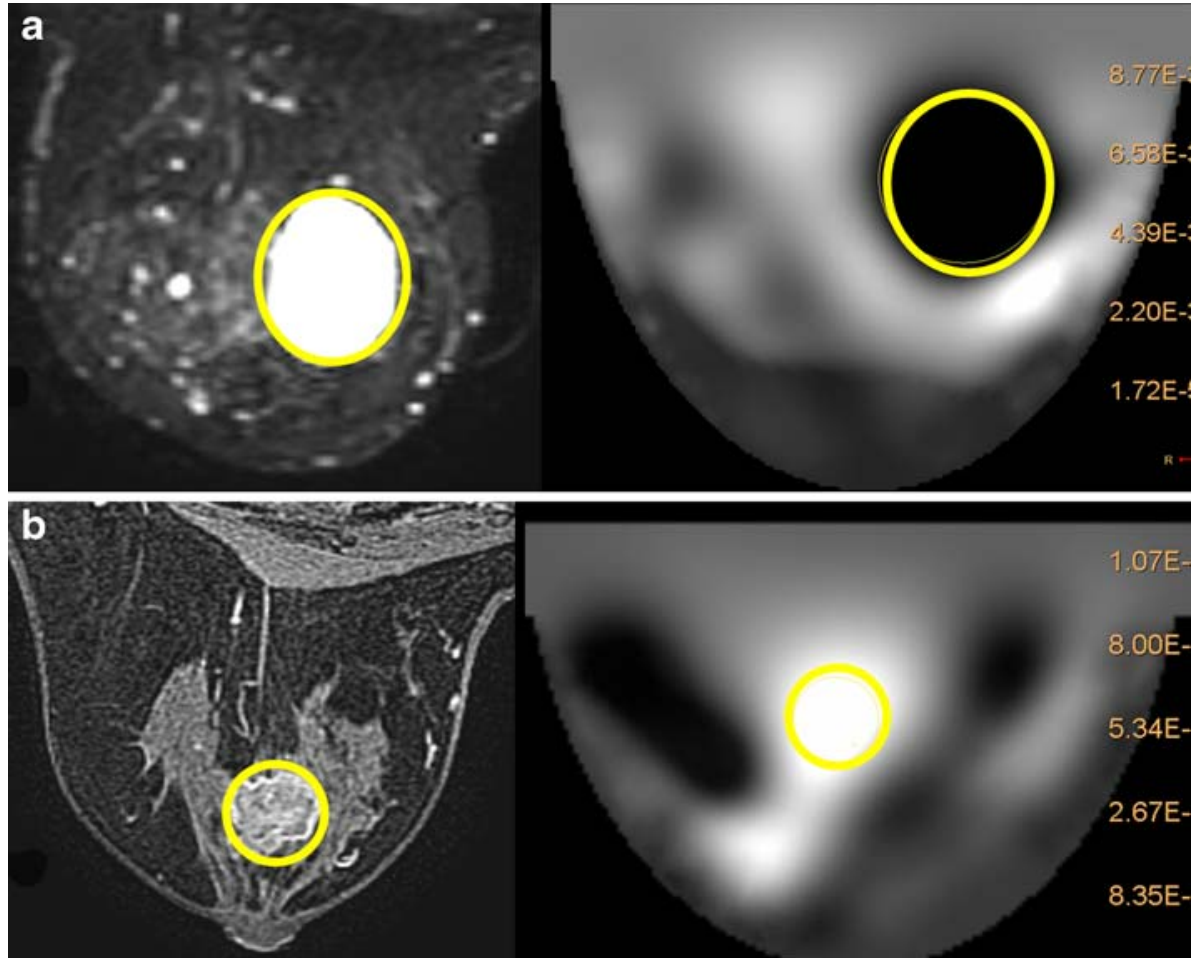
Table 1 Median (range) of quantitative score according to lesion presence and lesion type

\begin{tabular}{lllll}
\hline Wavelength $(\mathrm{nm})$ & \multicolumn{2}{l}{ Lesion type } & No lesion $(n=18)$ & \\
\cline { 2 - 4 } & Malignant $(n=10)$ & Fibroadenoma $(n=2)$ & Cyst $(n=6)$ & $1.39(0.32-3.21)$ \\
690 & $3.03(1.91-3.40)$ & $1.75(1.36-2.14)$ & $0.23(0.10-0.73)$ & $1.34(0.29-3.18)$ \\
730 & $2.94(1.74-6.31)$ & $1.50(1.01-1.98)$ & $0.17(0.07-0.75)$ & $1.29(0.72-2.58)$ \\
780 & $2.57(1.54-3.22)$ & $1.36(1.13-1.58)$ & $0.13(0.08-0.82)$ & $1.16(0.71-2.34)$ \\
\hline 50 & $2.15(1.27-2.55)$ & $1.30(1.24-1.35)$ & $0.13(0.08-0.89)$ & \\
\hline
\end{tabular}

these were excluded due to (a) technical limitations of our DOT system, i.e., leakage of matching fluid from the system (6 patients) and the inability to measure lesions located close to the patient's chest wall due to the geometry of the cup ( 8 patients); and (b) the inability to undergo DCE-MRI for reason of claustrophobia (3 patients) and physical limitations (2 patients did not fit in the MRI bore). The protocol was approved by the ethics committee of the University Medical Center Utrecht, and written informed consent was obtained from all patients.

\section{Optical imaging}

DOT was performed on a Philips diffuse optical tomography system (Philips Healthcare, Best, The Netherlands). A patient was placed in the prone position on the system bed with her breast suspended in a cup on which 507 optical fibers are mounted (Fig. 1). The 253 source fibers are connected to four continuous wave solid-state lasers (wavelengths 690, 730, 780, and $850 \mathrm{~nm})$ and interleaved with 254 detector fibers. The cup was filled with a matching fluid, which has optical properties similar to an average breast, to enable a stable optical coupling between fibers and breast, and to eliminate optical shortcuts around the breast. During imaging, the breast was sequentially illuminated from all source positions and light emanating from the breast was detected in parallel for each source position. Acquisition duration was approximately $1 \mathrm{~min}$ per wavelength per breast, leading to a total examination time of approximately 10 min per patient. After optical data acquisition, three-dimensional absorption images were reconstructed

Table 2 Discriminatory value of quantitative score for presence of malignancy $(n=10$; total of 36 breasts)

\begin{tabular}{ll}
\hline Wavelength $(\mathrm{nm})$ & $\begin{array}{l}\text { Area under ROC curve } \\
(95 \% \text { confidence interval })\end{array}$ \\
\hline 690 & $0.93(0.86-1.00)$ \\
730 & $0.92(0.85-1.00)$ \\
780 & $0.95(0.88-1.00)$ \\
850 & $0.93(0.86-1.00)$ \\
\hline
\end{tabular}

for each wavelength by using a linear reconstruction algorithm based on the Rytov approximation [9].

\section{MRI}

Dynamic contrast-enhanced breast MRI was performed on a 3.0-T clinical MR system (3.0T Achieva, Philips Healthcare, Best, The Netherlands). Patients were placed in prone position on a dedicated four-element SENSEcompatible phased-array bilateral breast coil (MRI devices, Würzburg, Germany) utilized for simultaneous imaging of both breasts. The MR protocol included an axial highresolution T1-weighted fast gradient echo (HR-T1FFE) fatsuppressed series (TE/TR 1.7/4.5 ms; inversion delay SPAIR $130 \mathrm{~ms}$; flip angle $10^{\circ}$; FOV $340 \times 340 \mathrm{~mm}^{2}$, acquired voxel size $0.66 \times 0.66 \times 1.6 \mathrm{~mm}^{3}$, reconstructed voxel size $\left.0.66 \times 0.66 \times 0.80 \mathrm{~mm}^{3}\right)$, followed by an axial T2-weighted fat-suppressed series (TE/TR 120/9,022 ms; inversion delay SPAIR $125 \mathrm{~ms}$; flip angle $90^{\circ}$; FOV $340 \times$ $340 \mathrm{~mm}^{2}$, acquired voxel size $1.01 \times 1.31 \times 2.0 \mathrm{~mm}^{3}$, reconstructed voxel size $0.66 \times 0.66 \times 2.00 \mathrm{~mm}^{3}$ ). Finally, dynamic contrast-enhanced fat-suppressed $\mathrm{T} 1$-weighted images were acquired (TE/TR 1.3/3.4 ms; flip angle $10^{\circ}$; FOV $320 \mathrm{~mm} \times 320 \mathrm{~mm}$, acquired voxel size $0.91 \mathrm{~mm} \times$ $0.91 \mathrm{~mm} \times 2.00 \mathrm{~mm}$, reconstructed voxel size $0.83 \mathrm{~mm} \times$ $0.83 \mathrm{~mm} \times 1.00 \mathrm{~mm}$, temporal resolution $50 \mathrm{~s}$ per dynamic acquisition) with a total of six dynamic acquisitions, one obtained before, and five obtained $0,60,120,180$, and $240 \mathrm{~s}$ after administration of a bolus injection of $0.1 \mathrm{mmol} /$ $\mathrm{kg}$ gadolinium-based contrast agent (Magnevist, Schering, Berlin, Germany) followed by a 20-ml saline flush at an injection rate of $3 \mathrm{ml} / \mathrm{s}$ with an automatic injector.

\section{Image interpretation}

MR images were interpreted by two breast radiologists with more than 10 years of experience and were used to derive the location of the lesions. A region of interest (ROI) was drawn at the lesion site location for all four optical absorption images (e.g., see Fig. 2). For comparison, a similar ROI was drawn at the mirror image lesion site location of the contralateral breast, where no lesion was found. The visibility of the lesions on 
Table 3 Intraobserver agreement for visibility scores (total of 36 breasts, four wavelengths per breast)

\begin{tabular}{lllll}
\hline Wavelength & \multicolumn{2}{l}{ Intraclass correlation coefficient $^{\mathrm{a}}(95 \%$ confidence interval $)$} & \multicolumn{2}{l}{ Kappa statistic $^{\mathrm{b}}(95 \%$ confidence interval $)$} \\
\cline { 2 - 3 } & Observer 1 & Observer 2 & Observer 1 & $0.88(0.82-0.94)$ \\
\hline All & $0.978(0.970-0.983)$ & $0.987(0.982-0.990)$ & $0.88(0.82-0.94)$
\end{tabular}

${ }^{a}$ Original visibility scores; one-way random effects model where people effects are random (single measures)

${ }^{\mathrm{b}}$ Visibility scores recoded into categories $(<-1,-1$ to $1,>1)$

DOT was assessed both quantitatively and qualitatively. Quantitative values were computed from the volume images of the optical absorption coefficient obtained from the DOT system. The mean absorption coefficient of the ROI was divided by the mean absorption of the background, which included the rest of the breast on that slice except for the lesion (the quantitative score). Qualitative scores for contrast relative to background were given independently by two readers for every ROI, on a scale from -4 to 4 , where: $0=$ no visibility; $1=$ slight heterogeneity seen at the site of the known lesion; $2=$ moderate contrast, but less/more than other structures, seen at the site of the known lesion; $3=$ contrast at the known lesion site comparable to that of other structures; $4=$ major contrast at the known lesion site; a minus sign was used if the signal at the ROI was lower than the background, and a plus sign when it was higher. To learn how to score the images, readers were shown an example set of classified images (not from the study population) before they started the scoring process. All images were made anonymously, placed in random order, and scored by two readers separately, without knowledge from other examinations. Images were scored again after 3 months in a second independent reading by the two investigators.

The reference standard for final diagnosis of the solid lesions was histopathology, whereas for the benign cysts and the healthy contralateral breast (mirror image) the reference standard was MRI. The patients diagnosed with benign cysts received a follow-up mammography and ultrasound after 6 months.

\section{Statistics}

Intra- and interobserver agreements between the two readers were calculated using kappa statistics and intraclass correlation coefficients [10]. Discriminatory values for presence of malignancy were determined by receiver operating characteristic (ROC) analyses. Cancer detection rates were calculated using a qualitative score of $\geq 2$ as a cutoff. The package SPSS 15.0 (SPSS Inc., Chicago, IL, USA) was used for all statistical computations.

\section{Results}

Seventeen patients (mean age 54, range 22-85) with 18 breast lesions underwent optical imaging and DCE-MRI. Of the 18 lesions, 10 lesions were diagnosed as malignant by histopathology after surgery (9 invasive ductal carcinomas and 1 invasive lobular carcinoma; final BI-RADS score was BI-RADS 5 in 7 lesions and BI-RADS 4 in 3 lesions) with a median diameter of $23.5 \mathrm{~mm}$ (range 13$54 \mathrm{~mm}$ ); 2 lesions were confirmed to be benign fibroadenomas (final BI-RADS score 3) by large core needle biopsy, with diameters of 13 and $24 \mathrm{~mm}$; and 6 lesions were diagnosed as benign cysts (final BI-RADS score 2) by ultrasound and MRI, with a median diameter of $28.5 \mathrm{~mm}$ (range $20-40 \mathrm{~mm}$ ); the 23 mirror image regions of the contralateral breasts appeared as normal breast tissue without lesions on DCE-MRI.

Quantitative scores (ROI-to-background ratios) are shown in Table 1. Scores for malignant lesions were higher (2.15-3.03) than those for fibroadenomas (1.30$1.75)$, cysts $(0.13-0.23)$, and the contralateral normal breast (1.16-1.39). Discriminatory values of quantitative scores for presence of malignancy are shown in Table 2, with areas under the ROC curves of 0.92-0.93.

For intraobserver agreements, intraclass correlation coefficients and kappa statistics were excellent (Table 3). Intraclass correlation coefficients were excellent and kappa statistics were good to excellent for the interobserver

Table 4 Interobserver agreement for visibility scores (total of 36 breasts, four wavelengths per breast)

\begin{tabular}{lll}
\hline Wavelength $(\mathrm{nm})$ & Intraclass correlation coefficient $^{\mathrm{a}}(95 \% \text { confidence interval })^{-}$ & Kappa statistic $^{\mathrm{b}}(95 \%$ confidence interval $)$ \\
\hline 690 & $0.96(0.92-0.98)$ & $0.77(0.58-0.96)$ \\
730 & $0.98(0.95-0.99)$ & $0.95(0.85-1.00)$ \\
780 & $0.96(0.92-0.98)$ & $0.80(0.61-0.99)$ \\
850 & $0.97(0.95-0.99)$ & $0.89(0.74-1.00)$ \\
\hline
\end{tabular}

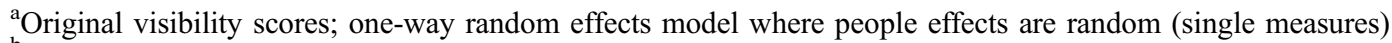

${ }^{\mathrm{b}}$ Visibility scores recoded into categories $(<-1,-1$ to $1,>1)$ 
Table 5 Median (range) visibility score according to lesion presence and lesion type

\begin{tabular}{|c|c|c|c|c|c|c|c|c|}
\hline \multirow[t]{3}{*}{ Wavelength (nm) } & \multicolumn{6}{|l|}{ Lesion type } & \multirow{2}{*}{\multicolumn{2}{|c|}{ No lesion $(n=18)$}} \\
\hline & \multicolumn{2}{|c|}{ Malignant $(n=10)$} & \multicolumn{2}{|c|}{ Fibroadenoma $(n=2)$} & \multicolumn{2}{|l|}{ Cyst $(n=6)$} & & \\
\hline & Observer 1 & Observer 2 & Observer 1 & Observer 2 & Observer 1 & Observer 2 & Observer 1 & Observer 2 \\
\hline 690 & $2(0-4)$ & $2(0-4)$ & $1(0-2)$ & $2(0-4)$ & $-4(-4$ to -1$)$ & $-4(-4$ to -1$)$ & $0(-2$ to 2$)$ & $0(0-3)$ \\
\hline 730 & $2(0-4)$ & $2(0-4)$ & $1(1-1)$ & $0(0-0)$ & $-4(-4$ to 0$)$ & $-4(-4$ to 0$)$ & $0(0-2)$ & $0(0-3)$ \\
\hline 780 & $2(0-4)$ & $4(0-4)$ & $0(0-0)$ & $0(0-0)$ & $-4(-4$ to -1$)$ & $-4(-4$ to 0$)$ & $0(-1$ to 1$)$ & $0(-2$ to 2$)$ \\
\hline 850 & $2(0-4)$ & $2(0-4)$ & $0(0-0)$ & $0(0-0)$ & $-4(-4$ to 0$)$ & $-4(-4$ to 0$)$ & $0(-1$ to 1$)$ & $0(0-2)$ \\
\hline
\end{tabular}

agreement (Table 4). Qualitative visibility scores are shown in Table 5. Scores for malignant lesions were higher (2-4) than for fibroadenomas $(0-2)$, cysts $(-4)$, and the contralateral normal breast $(0)$. Table 6 shows the discriminatory values of qualitative scores for presence of malignancy for both observers (equal results), with areas under the ROC curves of $0.97-0.99$. Cancer detection rates for the four wavelengths $(690,730,780$, and $850 \mathrm{~nm}$, respectively) were $70 \%, 80 \%, 80 \%$, and $70 \%$ for observer 1 ; and $60 \%$, $70 \%, 70 \%$, and $60 \%$ for observer 2 . Between $0 \%$ and $22 \%$ false positive results were found per wavelength for both observers.

\section{Discussion}

This study describes the first diagnostic evaluation under optimal settings of a new prototype DOT system with MRI as a benchmark in patients with breast lesions. Using a known lesion position, malignant lesions could be discriminated from benign tissue both quantitatively and qualitatively, mainly owing to higher hemoglobin contents causing more light absorption. Intra- and interobserver agreement were good to excellent, indicating a reproducible method.

Our results are in agreement with previously published studies using different techniques [11-26]. Detection rates ranging from 0.04 to 1.00 were reported, irrespective of lesion classification (benign/malignant). Cancer detection rates in our study were between $60 \%$ and $80 \%$ for each wavelength separately, using a cutoff value of 2 ; detection

Table 6 Discriminatory value of visibility score for presence of malignancy $(n=10$; total of 36 breasts)

\begin{tabular}{ll}
\hline $\begin{array}{l}\text { Wavelength } \\
(\mathrm{nm})\end{array}$ & $\begin{array}{l}\text { Area under ROC curve (95\% confidence interval) } \\
\text { Observer } 1 \text { and } 2\end{array}$ \\
\hline 690 & $0.97(0.89-1.00)$ \\
730 & $0.99(0.95-1.00)$ \\
780 & $0.97(0.89-1.00)$ \\
850 & $0.99(0.95-1.00)$ \\
\hline
\end{tabular}

rates and false positive results can likely be improved when combining information from four different wavelengths in one model (spectroscopic imaging). Similar to other research groups, we used knowledge of lesion localization from a reference standard (MRI in this instance) for the evaluation of the optical data. This approach in image interpretation may have resulted in limited false positive findings with consequent overestimation of the ROC analyses. However, the areas under the ROC curves justify further evaluation and development of the technique.

As this study represents the first evaluation of a new prototype for malignant lesion detection conclusions should be drawn with care due to important limitations of the system. In addition, because the sample size of the study was small and the study group was selective, obtained results cannot be extrapolated directly to a clinical diagnostic or screening setting. Technical limitations of the DOT system resulted in the exclusion of 14 of the 36 patients for image analysis. These major limitations comprised the leakage of matching fluid from the system resulting in large artifacts, and the limitation in the current geometry of the system to measure lesions located close to the patient's chest wall. Most likely, these lesions were physically located too far above the upper optical fibers in the cup to influence the light pathways during optical imaging. Improvement in cup shape is feasible and should be realized to visualize these lesions. A limitation of DOT in general is the poor spatial resolution $[1,16]$. This results in a lower signal-to-noise ratio and limited detection of very small lesions. Median diameters of the included lesions in our study were relatively long; the smallest detected lesion by DOT in our study sample was $13 \mathrm{~mm}$, and may therefore not be representative for the routine clinical and screening situation. Further studies including more patients with smaller lesions will be performed to get a more realistic estimate of the system's diagnostic value. A molecular imaging approach using certain fluorescent probes may vastly improve signal-to-noise ratios by signal amplification [27]. Target-specific fluorescent probes for optical imaging have already been used successfully in animal experiments [28-30].

In conclusion, this novel DOT system has the potential to discriminate malignant from benign breast tissue by assessing optical properties of the tissue in a reproducible 
quantitative and qualitative way. Important technical improvements are required before this technique will be ready for use in clinical practice.
Open Access This article is distributed under the terms of the Creative Commons Attribution Noncommercial License which permits any noncommercial use, distribution, and reproduction in any medium, provided the original author(s) and source are credited.

\section{References}

1. Gibson AP, Hebden JC, Arridge SR (2005) Recent advances in diffuse optical imaging. Phys Med Biol 50:R1-R43

2. Rice A, Quinn CM (2002) Angiogenesis, thrombospondin, and ductal carcinoma in situ of the breast. J Clin Pathol 55:569-574

3. Vaupel P, Harrison L (2004) Tumor hypoxia: causative factors, compensatory mechanisms, and cellular response. Oncologist 9(Suppl 5):4-9

4. Peters NH, Borel RI, Zuithoff NP et al (2008) Meta-analysis of MR imaging in the diagnosis of breast lesions. Radiology 246:116-124

5. Raza S, Chikarmane SA, Neilsen SS et al (2008) BI-RADS 3, 4, and 5 lesions: Value of US in Management-followup and outcome. Radiology 248:773781

6. Pisano ED, Gatsonis C, Hendrick E et al (2005) Diagnostic performance of digital versus film mammography for breast-cancer screening. N Engl J Med 353:1773-1783

7. Yeh E, Slanetz P, Kopans DB et al (2005) Prospective comparison of mammography, sonography, and MRI in patients undergoing neoadjuvant chemotherapy for palpable breast cancer. Am J Roentgenol 184:868-877

8. Leff DR, Warren OJ, Enfield LC et al (2008) Diffuse optical imaging of the healthy and diseased breast: A systematic review. Breast Cancer Res Treat 108:9-22

9. Nielsen T, Brendel B, Ziegler R et al (2008) Linear image reconstruction for a diffuse optical mammography system in a non-compressed geometry using scattering fluid. Biomedical optics. Optical Society of America, BMD23. http:/www.opticsinfobase.org/abstract. cfm?URI=BIOMED-2008-BMD23. Accessed 16 Mar 2008

10. Brennan P, Silman A (1992) Statistical methods for assessing observer variability in clinical measures. Br Med J (Clin Res Ed) 304:1491-1494
11. Chance B, Nioka S, Zhang J et al (2005) Breast cancer detection based on incremental biochemical and physiological properties of breast cancers: a six-year, two-site study. Acad Radiol 12:925-933

12. Floery D, Helbich TH, Riedl CC et al (2005) Characterization of benign and malignant breast lesions with computed tomography laser mammography (CTLM): initial experience. Invest Radiol 40:328-335

13. Franceschini MA, Moesta KT, Fantini $S$ et al (1997) Frequency-domain techniques enhance optical mammography: initial clinical results. Proc Natl Acad Sci USA 94:6468-6473

14. Gotz L, Heywang-Kobrunner SH, Schutz O et al (1998) Optical mammography in preoperative patients. Aktuelle Radiol 8:31-33

15. Gu X, Zhang Q, Bartlett $M$ et al (2004) Differentiation of cysts from solid tumors in the breast with diffuse optical tomography. Acad Radiol 11:53-60

16. Hsiang D, Shah N, Yu H et al (2005) Coregistration of dynamic contrast enhanced MRI and broadband diffuse optical spectroscopy for characterizing breast cancer. Technol Cancer Res Treat 4:549-558

17. Intes X (2005) Time-domain optical mammography SoftScan: initial results. Acad Radiol 12:934-947

18. Ntziachristos V, Yodh AG, Schnall MD et al (2002) MRI-guided diffuse optical spectroscopy of malignant and benign breast lesions. Neoplasia 4:347-354

19. Rinneberg H, Grosenick D, Moesta KT et al (2005) Scanning time-domain optical mammography: detection and characterization of breast tumors in vivo. Technol Cancer Res Treat 4:483496

20. Taroni P, Torricelli A, Spinelli L et al (2005) Time-resolved optical mammography between 637 and $985 \mathrm{~nm}$ : clinical study on the detection and identification of breast lesions. Phys Med Biol 50:2469-2488
21. Tomandl B, Doinghaus K Schulz-Wendtland R (1995) Laser mammography with near-infrared light. Rontgenpraxis 48:197-201

22. Yates T, Hebden JC, Gibson A et al (2005) Optical tomography of the breast using a multi-channel timeresolved imager. Phys Med Biol 50:2503-2517

23. Zhu Q, Cronin EB, Currier AA et al (2005) Benign versus malignant breast masses: optical differentiation with USguided optical imaging reconstruction. Radiology 237:57-66

24. Cerussi A, Hsiang D, Shah $N$ et al (2007) Predicting response to breast cancer neoadjuvant chemotherapy using diffuse optical spectroscopy. Proc Natl Acad Sci USA 104:4014-4019

25. Cerussi A, Shah N, Hsiang D et al (2006) In vivo absorption, scattering, and physiologic properties of 58 malignant breast tumors determined by broadband diffuse optical spectroscopy. J Biomed Opt 11:044005

26. Durduran T, Choe R, Yu G et al (2005) Diffuse optical measurement of blood flow in breast tumors. Opt Lett 30:2915-2917

27. Mahmood U, Weissleder R (2003) Near-infrared optical imaging of proteases in cancer. Mol Cancer Ther 2:489-496

28. Bremer C, Ntziachristos V, Weitkamp $\mathrm{B}$ et al (2005) Optical imaging of spontaneous breast tumors using protease sensing 'smart' optical probes. Invest Radiol 40:321-327

29. Hilger I, Leistner Y, Berndt A et al (2004) Near-infrared fluorescence imaging of HER-2 protein over-expression in tumour cells. Eur Radiol 14:1124-1129

30. Kumar SR, Quinn TP, Deutscher SL (2007) Evaluation of an 111Inradiolabeled peptide as a targeting and imaging agent for ErbB-2 receptor expressing breast carcinomas. Clin Cancer Res 13:6070-6079 